\title{
El control de procesos industriales y su influencia en el mantenimiento
}

\author{
Aurelio Arbildo López \\ Universidad de Lima \\ Ingeniería Industrial n. ${ }^{\circ} 29,2011$, ISSN 1025-9929, pp. 35-49 \\ Recibido: 4 de mayo del 2011 / Aprobado: 12 de mayo del 2011
}

\begin{abstract}
RESUMEN: El control de procesos toma en cuenta la medición y el análisis de las variables que determinan el funcionamiento de un proceso así como la toma de decisiones y la ejecución de acciones de control para gobernar dicho proceso. Aun cuando el control del proceso se realice con fines netamente operativos, siempre es posible capturar y almacenar información, que puede ser eficientemente procesada con fines de mantenimiento, como es el caso del mantenimiento predictivo. Asimismo, se acostumbra instrumentar máquinas y equipos de proceso para adquirir datos exclusivos para estos fines. Como una opción más, se pueden automatizar las tareas de mantenimiento.
\end{abstract}

Palabras clave: procesos industriales / mantenimiento de planta / control de proceso / mantenimiento automatizado

\section{Industrial process control and its influence on the maintenance}

ABstRaCT: The process control takes into account the measurement and the analysis of the variables, which define the behavior of the process, as well as the decision taking and actions to control such process. Even if the process control is meant for purely operational purposes, it is always possible to log and store information that can be efficiently processed for maintenance, as in the case of predictive maintenance. On the other side, we usually need instrumentation to acquire data exclusively for maintenance and, as a third option; the maintenance related tasks can also be automated.

Keywords: industrial processes / plan maintenance / process control / automated maintenance 


\section{INTRODUCCIÓN}

Toda planta industrial se diseña para llevar a cabo un proceso productivo que, partiendo de la materia prima y los insumos entrega bienes cuyo valor lo determina finalmente el mercado. Como el mundo globalizado impone un entorno de alta competencia, todas las empresas tratan de elevar su productividad y mejorar la calidad de los productos que ofrecen, haciendo el mejor uso posible de los recursos disponibles.

La mejor manera de mejorar la competitividad, utilizando la tecnología electrónica actual es mediante el control automático de los procesos. Esta tecnología está basada en la adquisición de datos que son extraídos directamente del proceso para ser analizados con el fin de tomar decisiones de control con un mínimo de intervención humana. El hecho de tener información directa del proceso es importante en términos de oportunidad, ya que tan pronto se produce un cambio, este es registrado y utilizado para mantener el proceso bajo control.

En todos los controles de procesos actuales se usan recursos de hardware y software disponibles en varias familias de controladores industriales de propósito general, que podemos clasificar tomando en cuenta los más utilizados. Así, tenemos: controladores lógicos programables (PLC), sistemas de control distribuido (DCS), computadores industriales (IPC) y controladores avanzados de procesos (PAC). Todos ellos han sido diseñados para el control industrial y preparados para que el usuario, con un mínimo de entrenamiento especializado, pueda programarlos.

En adición a estas familias se pueden también utilizar otras que requieren especialistas en programación, ya sea utilizando lenguajes como el ensamblador, lenguajes de alto nivel como el $\mathrm{C}$, u otros más especializados aún, como el VHDL. Desde que la mayoría de estos sistemas de control están basados en microprocesadores y cuentan con arquitectura de computador, siempre es posible tener acceso a la información que almacenan y que corresponde no solo a las variables que han sido captadas del proceso, como presiones, temperaturas, estados de máquinas y equipos, sino también las acciones que están en proceso o ya han sido realizadas. 
Figura 1

Controlador avanzado de procesos (PAC)

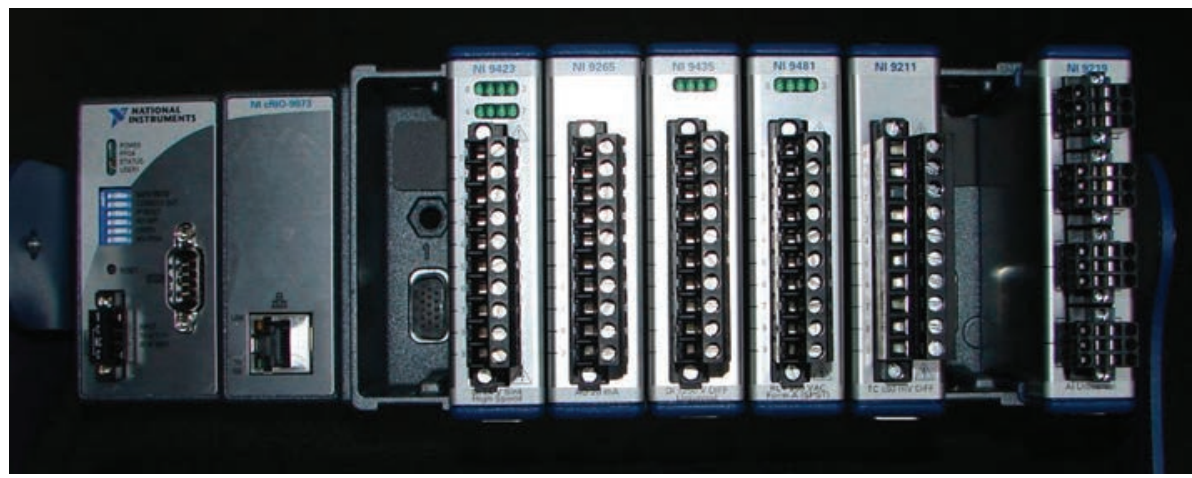

Foto del autor.

Típicamente, los controladores de procesos se conectan a computadoras de supervisión en las que se ejecuta el software encargado de la supervisión y el control, denominado "software Scada", que permite visualizar lo que está sucediendo en el proceso y a la vez controlarlo. Al tener ahora la información en un computador se abre una gama de posibilidades de uso de la información, entre las que destaca el control estadístico de procesos como herramienta poderosa tanto para el control de calidad como para el mantenimiento.

Por otro lado, la información carece de valor si no se utiliza, y se "deprecia" si es usada tarde; por ello, la tendencia es tener la información disponible para quien la vaya a utilizar dondequiera que se la requiera y en el momento en que se necesite. Esto se logra a través de un sistema integrado de información que consiste esencialmente en un sistema eficiente de comunicaciones (red de comunicaciones integrada desde el piso de planta hasta el máximo nivel gerencial), información muy bien organizada para que pueda ser compartida (base de conocimiento), y las herramientas de hardware y software para el uso adecuado de dicha información.

De esta manera, la información captada por los instrumentos de campo, la maquinaria, los equipos instrumentados y los sistemas de control terminan incorporados a la base de datos empresarial. El sistema de mantenimiento se incorpora como una función empresarial al sistema integrado de información y puede aprovechar óptimamente los 
recursos compartidos. En este orden de ideas el sistema de mantenimiento se nutre de los insumos disponibles aun cuando muchos de ellos no hayan sido originalmente captados para este fin.

\section{EL CONTROL DE PROCESOS Y SU RELACIÓN CON EL MANTENIMIENTO}

El control de procesos consiste en dos funciones claramente diferenciadas: la adquisición de datos y el control. Si tratamos de establecer el nexo con el mantenimiento concluiremos rápidamente que la adquisición de datos contribuye con la información para el mantenimiento y las acciones de control con la implantación de las acciones con fines tanto operativos como de mantenimiento. Desde que es mucho más ilustrativo ver los casos particulares en los que se hace evidente el valor del concepto, partiremos de ejemplos para luego generalizar dicho caso a todos los de su clase.

A manera de ensayo veamos el caso del control de calidad de hilo de nylon. La conclusión apresurada es: el control de calidad del hilo no tiene nada que ver con el mantenimiento; sin embargo, si hacemos la medición continua del diámetro del hilo podríamos encontrar, en tres escalas de tiempo diferentes, lo siguiente: a) variaciones del espesor en periodos cortos, variaciones que pueden ser resultado de la precisión de las mediciones, como también producto de vibraciones en las máquinas de proceso y, si este es el caso y la información es suficientemente detallada, un análisis de frecuencia puede dar luces sobre la máquina, que probablemente esté contribuyendo a esta variación; b) las variaciones en la siguiente escala de tiempo, en el caso de ser una variación periódica, normalmente está relacionada con alguna de las máquinas involucradas en el proceso, ya que los diámetros de las partes rotatorias por donde ha pasado el hilo originan variaciones que, en caso de desbalance, provocarán imperfecciones periódicas correlacionables con dicha máquina, por ello es fácilmente identificable dónde focalizar el mantenimiento, y c) la información de largo plazo permite identificar la tendencia de las imperfecciones; si la amplitud de las fluctuaciones de periodos cortos tiende a incrementarse en el largo plazo, indica que hay un aumento progresivo de las vibraciones, y si la amplitud de las fluctuaciones de periodos medios tiende a aumentar estaremos presenciando la progresión del desbalance. En ambos casos podemos fijar la máxima amplitud tolerable, que puede ser predicha con mucha antici- 
pación si proyectamos permanentemente la tendencia, apuntando claramente hacia lo que se conoce como mantenimiento predictivo.

\subsection{Extrayendo información útil para el mantenimiento}

\subsubsection{Del control de calidad}

En el párrafo anterior se ha descrito un ejemplo de cómo la información relativa al producto puede identificar las necesidades de mantenimiento de las maquinarias y equipos que la han producido. Esta es una primera categoría de la relación entre el control de procesos y el mantenimiento, y en particular muestra la relación estrecha que existe entre el control de calidad en línea y el mantenimiento.

Existen muchos casos que muestran esta relación, por ello me referiré a un ejemplo más para ilustrar este caso. Hace un tiempo (Arbildo 2000:15) desarrollamos un prototipo que permite medir el nivel de llenado de las botellas de agua carbonatada utilizando técnicas de visión artificial, es decir utilizando cámaras de vídeo y un computador para la captura y análisis de la imagen que posibilite la aceptación o el rechazo de las botellas luego del llenado. Como parte del prototipo se diseñó un esquema de medición sincronizada que permitía conocer la posición del carrusel donde había sido llenada la botella bajo inspección, de esta forma es posible hacer un histograma de frecuencias de los rechazos por nivel alto y bajo en función de la posición de llenado. Un pico en una posición dada para los rechazos por nivel alto implica que dicha posición tiene desgaste en el sello toda vez que el llenado se hace por balance de presión y una pérdida de presión debido al desgaste origina un sobrellenado en la botella; de esta forma, se logra identificar el lugar preciso donde se debe realizar el mantenimiento.

La información procedente del control de calidad a menudo está correlacionada con el funcionamiento de las máquinas, por ello es información útil con fines de mantenimiento.

\subsubsection{De la supervisión de los procesos}

Para la supervisión de la generación de energía se usan regularmente instrumentos de medición de variables eléctricas. Dichos medidores brindan información de voltajes, corrientes, potencia activa y reactiva, 
factor de potencia y otros; resulta entonces evidente que están orientados fundamentalmente a supervisar la generación y a contabilizar el producto (energía generada); sin embargo, si complementamos la medición con valores de consumo de combustible podemos calcular la eficiencia de cada unidad en términos de energía producida por unidad de combustible gastado. De esta forma, es posible operar las unidades más eficientes a bajas demandas, ingresando progresivamente las siguientes, y nuevamente la tendencia de la disminución de eficiencia permite programar la oportunidad del mantenimiento de tal o cual unidad.

Los sistemas de supervisión y control, también denominados sistemas Scada, colectan grandes cantidades de información con las cuales es posible crear indicadores a menudo relacionados con las máquinas y equipos de procesos. Muchas veces, los sistemas Scada son capaces de soportar la incorporación de rutinas de diagnóstico, sistemas experto compactos y herramientas de control estadístico, como diagramas de Pareto y otras muy utilizadas en mantenimiento.

\subsubsection{Del control de máquinas críticas}

Cuando una máquina o equipo es delicado en su funcionamiento, cuando su valor es elevado o el proceso requiere alta confiabilidad, es necesario incluir dispositivos de medición y control en las máquinas mismas. En esta categoría se incluye con bastante frecuencia la medición de temperaturas, ya que el sobrecalentamiento es un indicador inequívoco de funcionamiento inadecuado de la máquina o el equipo, o que está siendo sobreexigido y se deben tomar acciones de protección sobre dicho bien de capital. Al igual que las temperaturas se acostumbra también vigilar presiones, calidad del lubricante, vibraciones y cualquier otra variable directa o indirectamente relacionada con el buen funcionamiento del bien. Cuando los valores de las variables críticas de los equipos son colectados y analizados permanentemente, es posible ver las tendencias y predecir el momento en que debe hacerse el mantenimiento, por lo que los sistemas de supervisión contribuyen directamente al mantenimiento predictivo. En la figura 2 se muestra la pantalla de la computadora de supervisión en una planta de almacenamiento de gas; el compresor alimenta a las tuberías de almacenamiento de alta presión que permite guardar el exceso de gas entregado por el proveedor y que no ha sido consumido por los generadores de energía. Desde esta pantalla es posible monitorear permanentemente el funcionamiento del compresor. 
Figura 2

Supervisión de un compresor de gas en aplicación

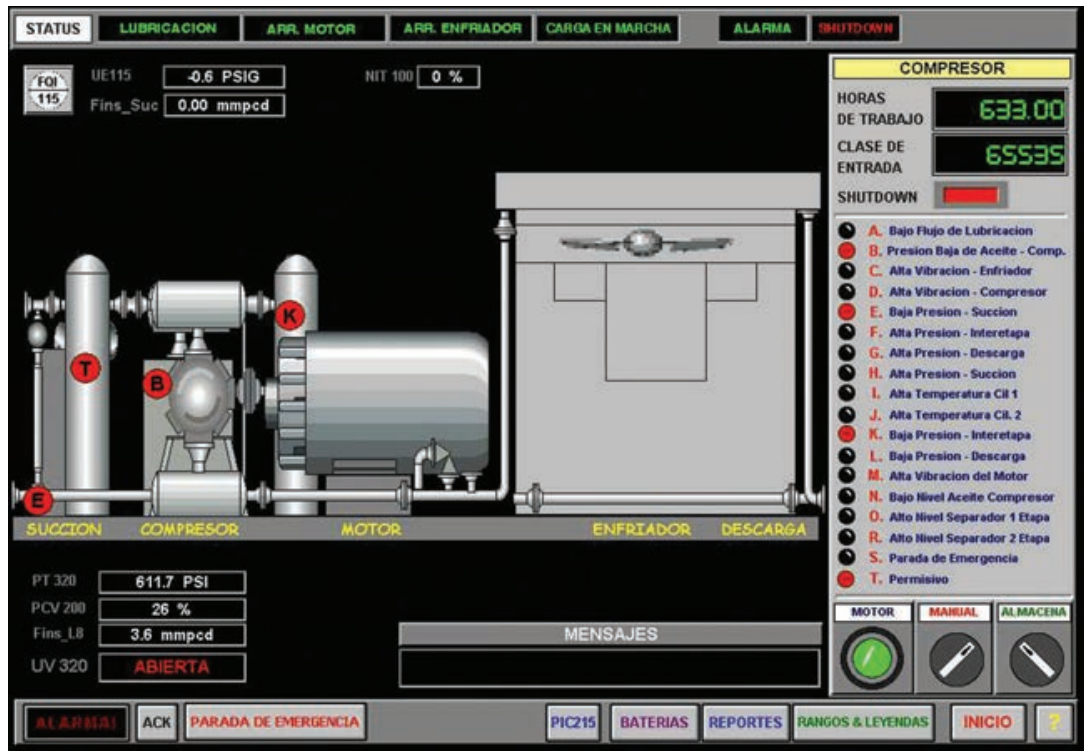

Fuente: Sociedad Inducontrol Ingeniería S.A.C. (2011).

Nuevamente, las máquinas que incorporan mediciones relativas a su funcionamiento permiten colectar información necesaria para su mantenimiento, así como la posibilidad de diagnóstico de fallas, envejecimiento y previsión de deterioro futuro.

\section{MANTENIMIENTO AUTOMATIZADO}

La tendencia actual es que aun las máquinas y los equipos simples tengan incorporados dispositivos basados en un microprocesador o un equivalente, para que de esta forma dichos equipos puedan ser conectados a sistemas más complejos de manejo de información. A medida que los equipos se vuelven más sofisticados y la electrónica asociada a la adquisición y manejo de información disminuye sus precios, es común que se incorporen rutinas de diagnóstico que permiten vigilar permanentemente el buen funcionamiento de dichos dispositivos.

Todo taller de mantenimiento está equipado con un mínimo de instrumentos para pruebas y medidas. Instrumentos como osciloscopios, analizadores de frecuencia y equipos de termografía vienen con opcio- 
nes de conexión a computadores, y, en los últimos años, están proliferando los llamados instrumentos virtuales. Un instrumento virtual es un verdadero instrumento cuya mayor diferencia es que el computador toma el lugar del instrumento, así, se requiere incorporar al computador actual las interfaces necesarias (osciloscopios, multímetros, analizadores de frecuencia, etcétera) para la adquisición de datos y el software que haga las funciones del instrumento con la ventaja que una sola interfaz puede eventualmente sustituir a varios instrumentos. La figura 3 es un ejemplo de instrumento virtual y corresponde a una imagen generada por el autor usando tarjeta osciloscopio DAQCard-5102 de National Instruments ${ }^{\mathrm{TM}}$ en un computador portátil PC con señales generadas externamente.

Figura 3

Instrumento virtual que muestra el espectro temporal, su respectiva componente de frecuencias y los controles del instrumento

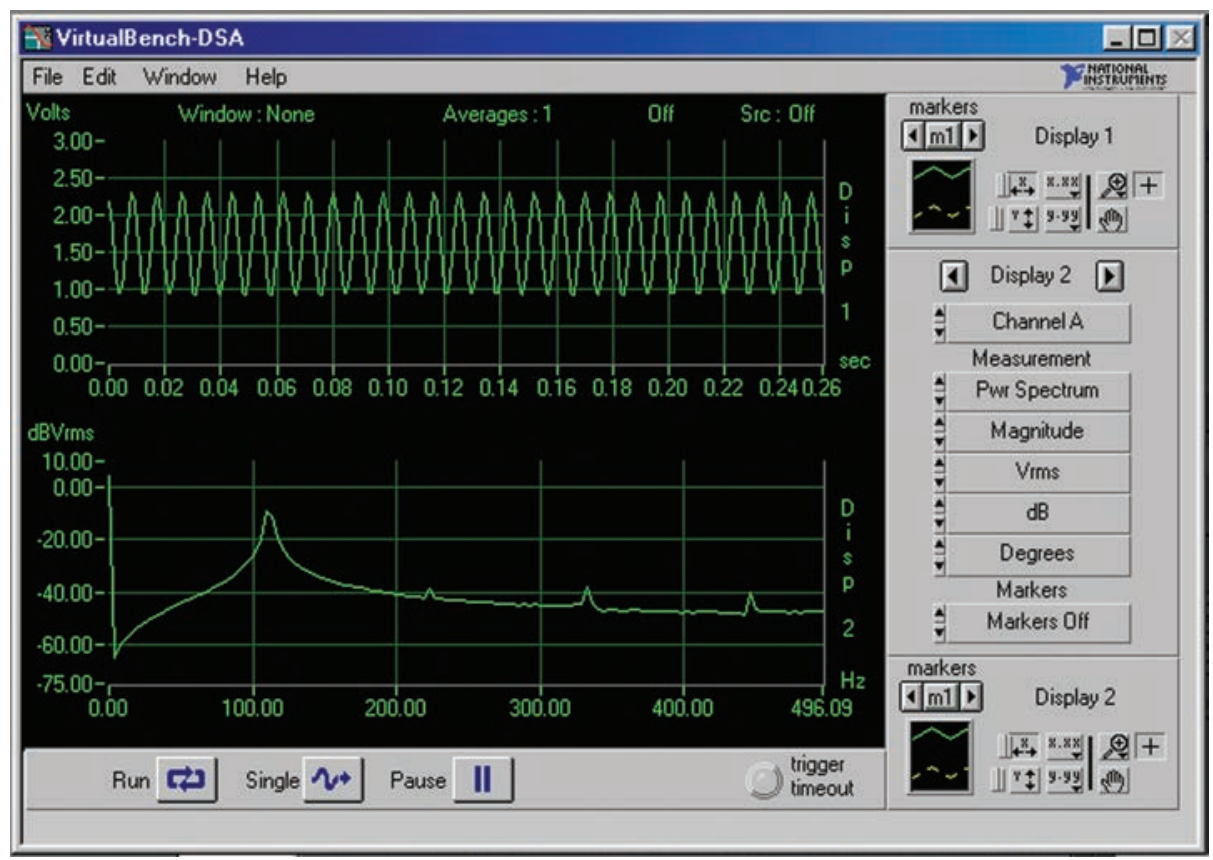

Captura de pantalla.

Elaboración propia. 
Cuando se tienen los instrumentos en el computador, es muy fácil automatizar las pruebas y medidas relativas al mantenimiento. $\mathrm{Al}$ ser los recursos del computador muy grandes, es posible almacenar datos históricos para comparación, especificaciones técnicas de referencia, protocolos de pruebas, fichas de mantenimiento y otras. La idea genérica del banco de pruebas automatizado es la de conectar el instrumento virtual a los puntos de prueba, enviar señales (tan sofisticadas como queramos) de estímulo, medir la respuesta y generar el diagnóstico con ayuda del software.

Los avances en la microelectrónica permiten que el laboratorio de pruebas sea portátil, de tal manera que el banco de pruebas viaje al campo con mucha facilidad y permita hacer el diagnóstico y mantenimiento en la planta misma o en el campo propiamente dicho.

Figura 4

Sistema de pruebas de cables para bombas sumergibles (permite identificar hilos fracturados y predecir el momento del cambio del cable)

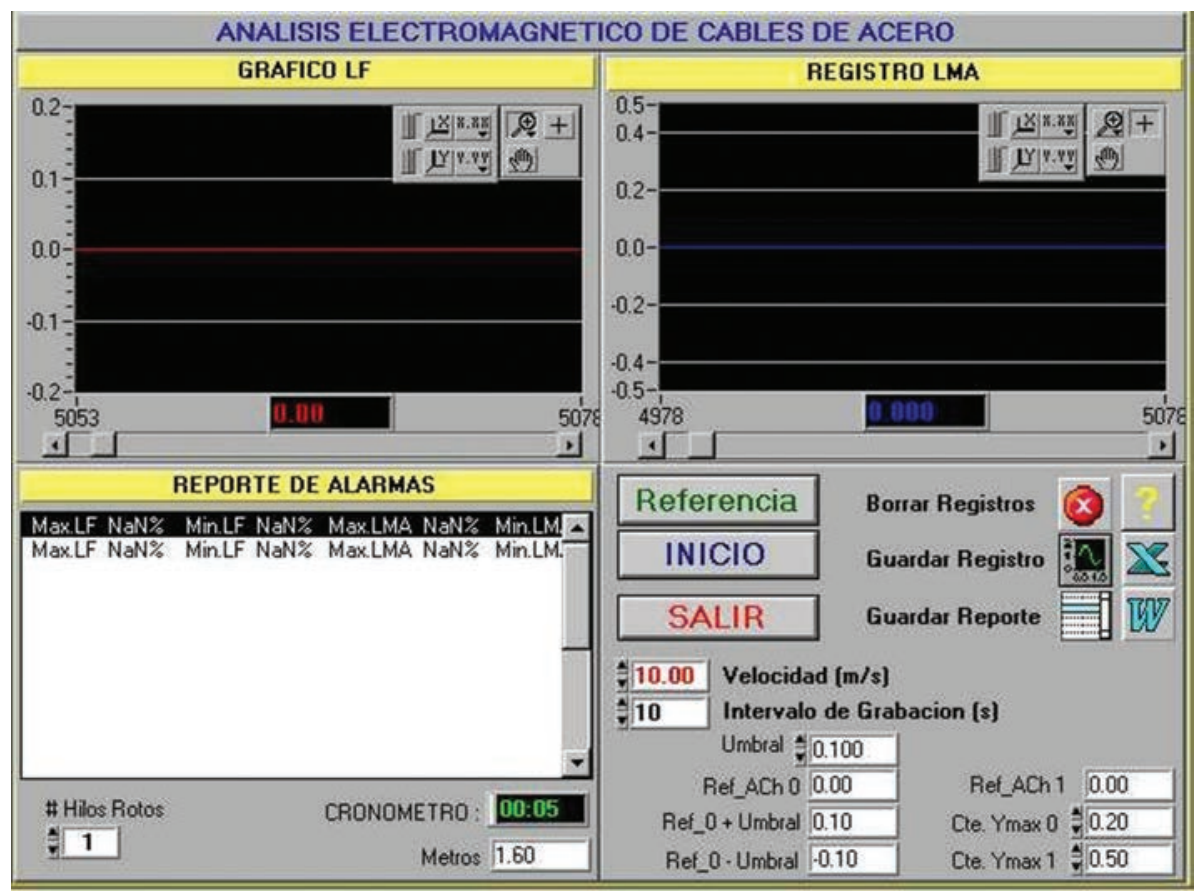

Fuente: Sociedad Inducontrol Ingeniería S.A.C. (2011). 
El laboratorio de pruebas automatizado viene a ser solo un componente del sistema de mantenimiento que comparte los recursos del sistema integrado de información en las empresas, las otras funciones de mantenimiento se nutren de la información y los recursos disponibles en dicho sistema integrado.

\section{LA SIMULACIÓN Y LA EXPERIMENTACIÓN ORIENTADA AL MANTENIMIENTO}

El conocimiento teórico del funcionamiento de los sistemas permite extraer información útil para el mantenimiento a partir de mediciones indirectamente relacionadas con el funcionamiento de dichos sistemas. Actualmente existe una gran variedad de herramientas de simulación así como de adquisición de datos y control tal que permiten simular, adquirir información y ejecutar las acciones de control, mantenimiento y optimización con diversos fines.

A manera de ejemplo veremos el caso de simulación y mediciones experimentales hechas para la identificación de fallas en motores, sobre la base del análisis de la corriente en las fases, cuyo detalle se encuentra en las memorias del Congreso Nacional de Ingeniería Mecánica, Eléctrica y Ramas Afines (Conimera) (Urcia et al. 1999).

En dicha oportunidad se realizó la simulación utilizando Matlab y mediciones en un motor experimental de la Universidad Nacional de Ingeniería y en la planta de Centromín Perú (posteriormente Doe Roe), utilizando un sistema de adquisición de datos con tarjeta multifunción para señales analógicas y digitales, así como el software LabView ${ }^{\left(\mathrm{TM}^{\circ}\right)}$ de National Instruments Co. USA.

Las mediciones consistieron en el registro de las corrientes en las tres fases, para construir el denominado vector de corriente de Park (VCP), que combina dicha información en un nuevo vector DQ y se basa en que la máquina de inducción trifásica puede ser estudiada fácilmente realizando una transformación lineal de los arrollamientos estatóricos fijos y arrollamientos rotóricos en movimiento, en arrollamientos estatóricos fijos y rotóricos pseudoestacionarios (modelo D-Q). 
Figura 5

Esquema del sistema experimental utilizado

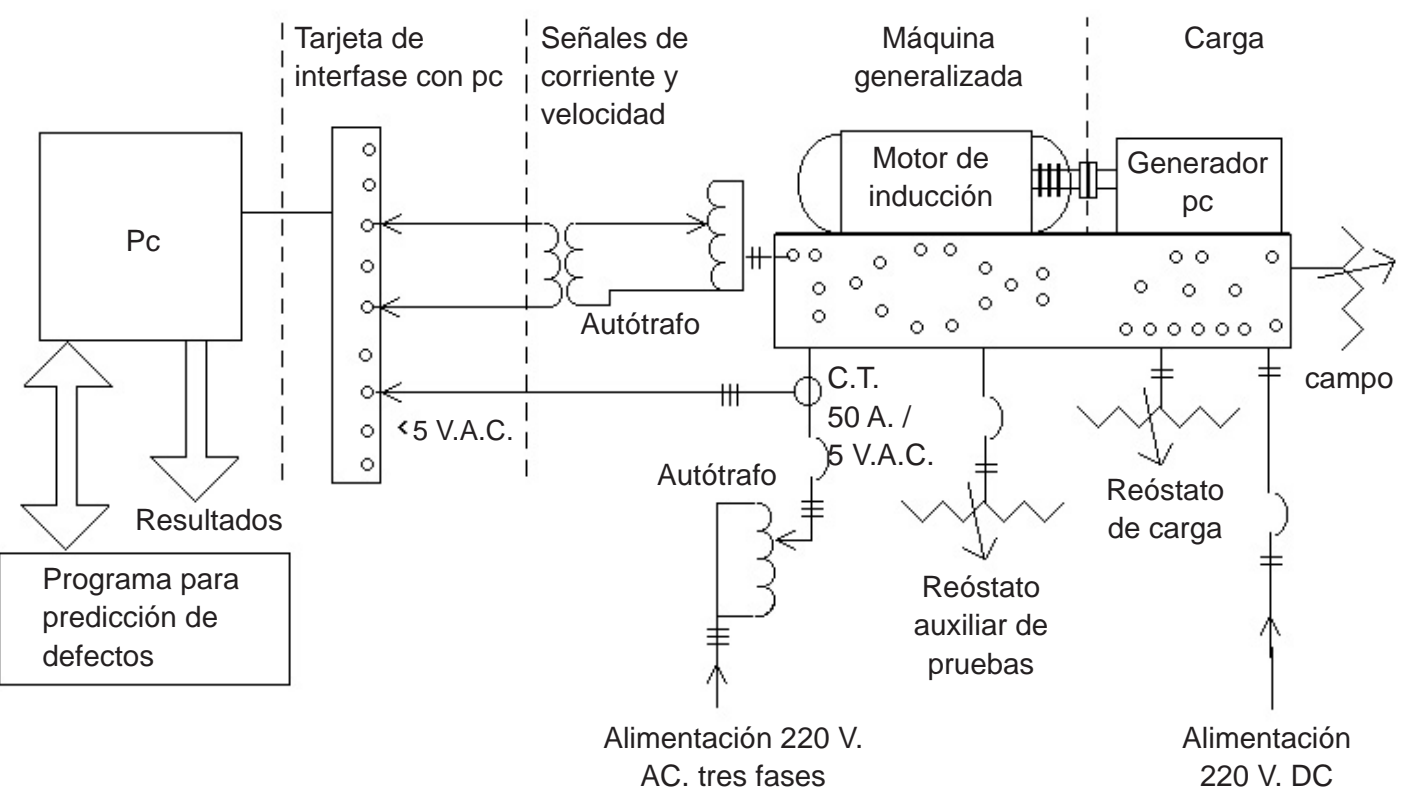

Fuente: Urcia et al. (1999).

En adición al análisis de las trayectorias DQ se hizo también un análisis espectral, y en el dominio del tiempo, para obtener información interesante sobre el comportamiento del motor ante fallas simuladas.

Como quiera que el artículo completo se encuentra publicado (Urcia et al. 1999), en este caso nos limitaremos a presentar algunos resultados gráficos que dan una idea de la aplicabilidad de esta metodología para el diagnóstico de fallas en motores. 
Figura 6

Análisis de la corriente durante el arranque

Transitorio corriente

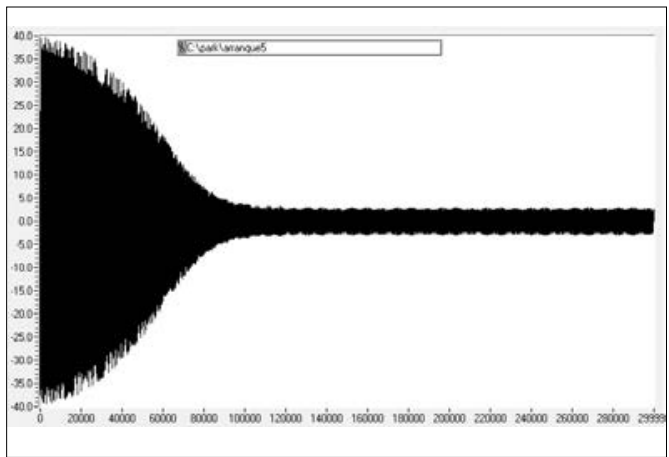

Vector de park

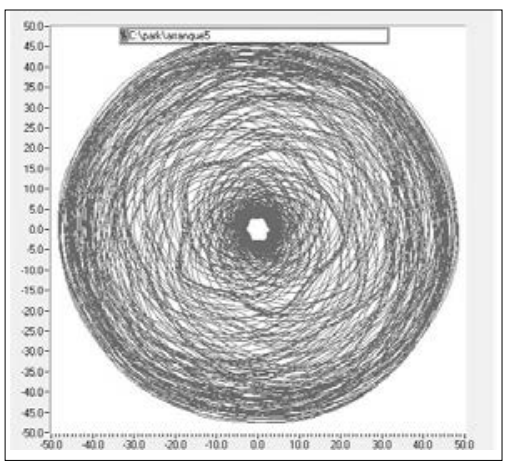

Fuente: Urcia (1999).

La figura 6 muestra el transitorio de la corriente de arranque y la correspondiente trayectoria DQ. Es interesante notar que la velocidad de los sistemas de adquisición de datos basados en computador permitió adquirir una cantidad impresionante de datos durante aproximadamente 8 segundos sin perder la componente en frecuencia de la corriente a razón de un dato por milisegundo.

En la figura 7 se muestra la medición de las corrientes en el dominio del tiempo, la frecuencia y las trayectorias del vector de corriente de Park (VCP) para simulación de fracturas de 1, 2 y 3 barras.

Podemos apreciar los resultados de la simulación, así como la medición experimental del motor sin defectos y el caso extremo de interrupción de bobinas que nos muestra claramente el efecto de fallas en estas y el esquema de conexiones para la simulación de cortocircuito en las espiras y su correspondiente efecto sobre las trayectorias VCP. 
Figura 7

Simulación de fractura de barras y mediciones correspondientes

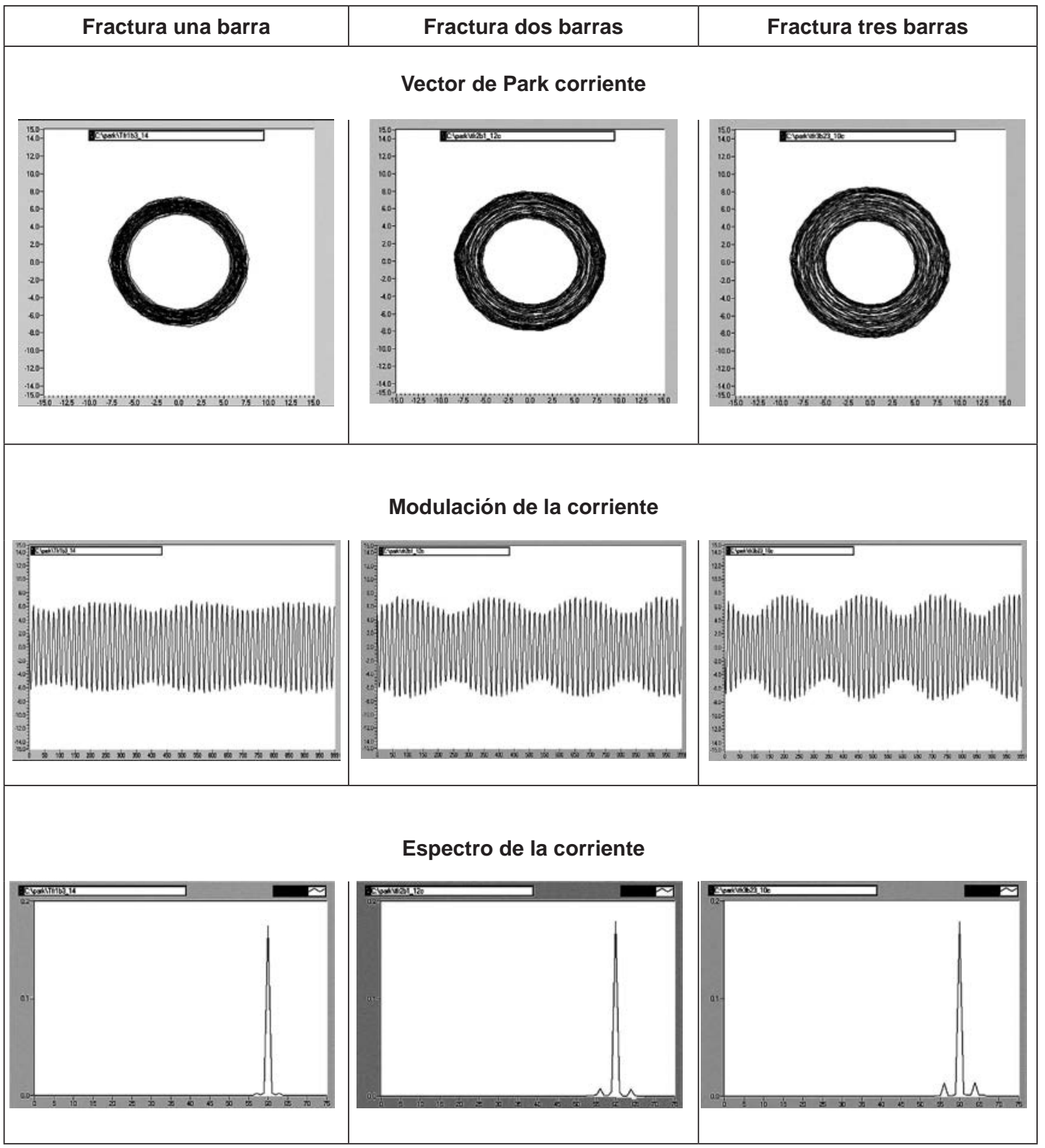

Fuente: Urcia et al. (1999). 


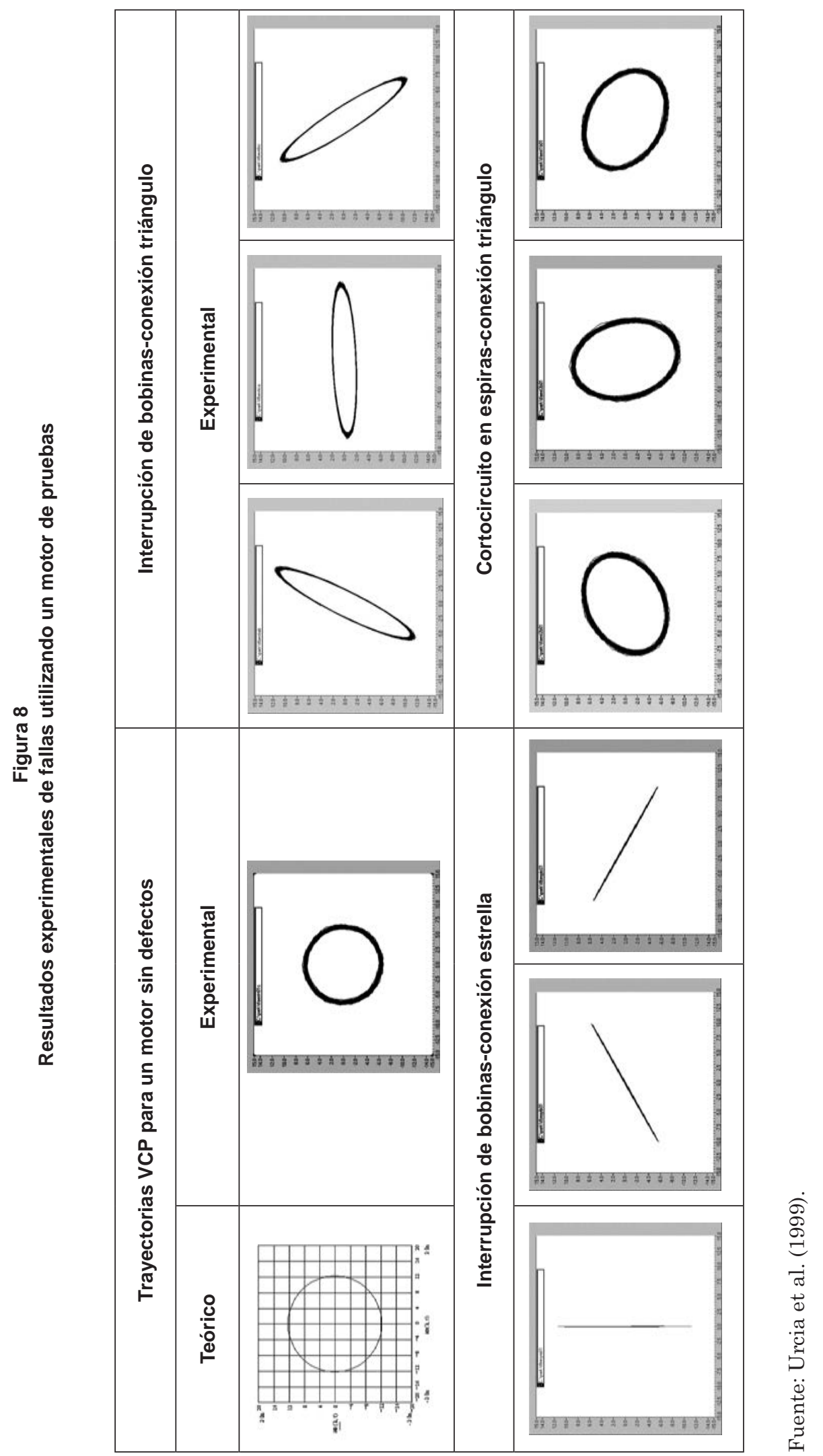




\section{CONCLUSIÓN}

Desde que el mantenimiento es un proceso controlable, todas las herramientas típicamente utilizadas para el control de procesos industriales, que incluye: los sistemas de adquisición de datos, los sistemas de control, el control supervisor (Scada), el control estadístico de procesos

y los sistemas integrados de información, son herramientas útiles para el sistema de mantenimiento.

\section{BIBLIOGRAFÍA}

Arbildo, Aurelio; Baca, Luis y Carlos Pezantes (2001). "Aplicaciones de control y supervisión de sistemas de generación y distribución eléctrica”. Industria al Día. Año 6, núm. 23. Lima: Grupo Editorial Contexto.

— (2000). "Procesamiento de imágenes para control de procesos". Industria al Día. Año 5, núm. 20. Lima: Grupo Editorial Contexto.

Sociedad Inducontrol Ingeniería SAC (2011). Archivo de Proyectos. Información autorizada. Lima.

Urcia, José; Ramírez, Roberto y Aurelio Arbildo (1999). "Metodologías de diagnóstico de fallas en el motor de inducción trifásico mediante el análisis de la corriente y su aplicación en la implementación de estrategias de mantenimiento predictivo". Documento presentado en el XIII Congreso Nacional de Ingeniería Mecánica, Eléctrica y Ramas Afines (Conimera), organizado por el Colegio de Ingenieros del Perú. Lima, 25-28 de agosto de 1999. 